\title{
MAPS PRESERVING PERIPHERAL SPECTRUM OF JORDAN PRODUCTS OF OPERATORS
}

\author{
JiANLIAN CUI AND CHI-KWONG Li
}

\begin{abstract}
Let $\mathscr{A}$ and $\mathscr{B}$ be (not necessarily unital or closed) standard operator algebras on complex Banach spaces $X$ and $Y$, respectively. For a bounded linear operator $A$ on $X$, the peripheral spectrum $\sigma_{\pi}(A)$ of $A$ is defined by $\sigma_{\pi}(A)=\left\{z \in \sigma(A):|z|=\max _{w \in \sigma(A)}|w|\right\}$, where $\sigma(A)$ denotes the spectrum of $A$. Assume that $\Phi: \mathscr{A} \rightarrow \mathscr{B}$ is a map and the range of $\Phi$ contains all operators with rank at most two. It is proved that the map $\Phi$ satisfies the condition that $\sigma_{\pi}(\Phi(A) \Phi(B)+\Phi(B) \Phi(A))=\sigma_{\pi}(A B+B A)$ for all $A, B \in \mathscr{A}$ if and only if either there exists an invertible operator $T \in \mathscr{B}(X, Y)$ such that $\Phi(A)=\varepsilon T A T^{-1}$ for every $A \in \mathscr{A}$; or $X$ and $Y$ are reflexive and there exists an invertible operator $T \in \mathscr{B}\left(X^{*}, Y\right)$ such that $\Phi(A)=\varepsilon T A^{*} T^{-1}$ for every $A \in \mathscr{A}$, where $\varepsilon \in\{1,-1\}$. Furthermore, the same conclusion holds if $\mathscr{A}$ and $\mathscr{B}$ are replaced by standard real Jordan algebras of self-adjoint operators on complex Hilbert spaces. If $X$ and $Y$ are complex Hilbert space, we characterize also maps preserving the peripheral spectrum of the product $A B^{*}+B^{*} A$, and prove that such maps are of the form $A \mapsto \gamma U A U^{*}$ or $A \mapsto \gamma U A^{t} U^{*}$, where $U \in \mathscr{B}(X, Y)$ is a unitary operator and $\gamma \in \mathbb{C}$ with $|\gamma|=1, A^{t}$ denotes the transpose of $A$ for an arbitrary but fixed orthonormal basis of $X$.
\end{abstract}

Mathematics subject classification (2010): 47B49, 47A12.

Keywords and phrases: Peripheral spectrum, Jordan products, Banach space, nonlinear preserver maps.

\section{REFERENCES}

[1] B. Aupetit, Spectrum-preserving linear mappings between Banach algebras or Jordan-Banach algebras, J. London Math. Soc. 62 (2000), 917-924.

[2] B. Aupetit AND DU T. Mouton H., Spectrum preserving linear mappings in Banach algebras, Studia Math. 109 (1994), 91-100.

[3] Z. F. Bai And J. C. Hou, Additive Maps Preserving Nilpotent Operators or Spectral Radius, Acta Mathematica Sinica 21, 5 (2005), 1167-1182.

[4] L. BARIBeAU And T. RAnSford, Non-linear spectrum-preserving maps, Bull. London Math. Soc. 32 (2000), 8-14.

[5] P. BotTA, S. PIERCE, AND W. WATKIns, Linear transformations that preserve the nilpotent matrices, Pacific J. Math. 104 (1983), 39-46.

[6] M. BREŠAR AND P. ŠEMRL, Linear maps preserving the spectral radius, J. Fun. Anal. 142 (1996), $360-368$.

[7] M. BREŠAR AND P. ŠEMRL, Invertibility preserving maps preserve idempotents, Michigan J. Math. 45 (1998), 483-488.

[8] J. L. CUi AND J. C. Hou, Additive maps on standard operator algebras preserving parts of the spectrum, J. Math. Anal. Appl. 282 (2003), 266-278.

[9] J. L. Cui And J. C. Hou, A Characterization of Homomorphisms Between Banach Algebras, Acta Math. Sinica 20, 4 (2004), 761-768.

[10] J. L. CUI AND J. C. HoU, Linear maps between Banach algebras compressing certain spectral functions, The Rocky Mountain Journal of Math. 34, 2 (2004), 565-584.

[11] S. P. DU, J. C. HoU, Z. F. BAI, Nonlinear maps preserving similarity on $\mathscr{B}(H)$, Linear Algebra Appl. 422 (2007), 506-516. 
[12] Y. Eidelman, V. Milman and A. Tsolominitis, Functional Analysis, An Introduction, American Mathematical Society, Providence, Rhode Island, 2004.

[13] L. A. HARRIS, R. V. KADISON, Affine mappings of invertible operators, Proc. Amer. Math. Soc. 124 (1996), 2415-2422.

[14] J. C. Hou, J. L. CUI, A Note on Nilpotency Preserving Additive Maps and Applications, Journal of Mathematical Study 38, 1 (2005), 1-9.

[15] J. C. HoU, K. HE AND X. ZHANG, Maps preserving numerical radius or cross norms of products of self-adjoint operators, Acta Math. Sinica, to appear.

[16] J. C. Hou, C. K. LI, N. C. WonG, Jordan isomorphisms and maps preserving spectra of certain operator products, Studia Math. 184 (2008), 31-47.

[17] J. C. Hou, C. K. LI, N. C. Wong, Maps Preserving the spectrum of generalized Jordan product of operators, Linear Algebra Appl. 432, 4 (2010), 1049-1069.

[18] A. A. JAFARIAN AND A. Sourour, Spectrum preserving linear maps, J. Funct. Anal. 66 (1986), 255-261.

[19] I. KAPlansky, Infinite Abelian groups, U. of Michigan Press, Ann Arbor, Michigan, 1954.

[20] S. LAMBERT, A. LutTMAN AND T. TONEV, Weakly peripherally-multiplicative mappings between uniform algebras, Contemp. Math. 435 (2007), 265-281.

[21] A. Luttman and T. Tonev, Uniform algebra isomorphisms and peripheral multiplicativity, Proc. Amer. Math. Soc. 135 (2007), 3589-3598.

[22] L. Molnár, Some characterizations of the automorphisms of $B(H)$ and $C(X)$, Proc. Amer. Math. Soc. 130 (2001), 111-120.

[23] M. TAKESHI AND H. DAI, A generalization of peripherally-multiplicative surjections between standard operator algebras, Central Eur. J. Math. 7, 3 (2009), 479-486.

[24] T. Tonev And A. LutTman, Algebra isomorphisms between standard operator algebras, Studia Math. 191 (2009), 163-170.

[25] U. UHLHORN, Representation of symmetry transformations in quantum mechanics, Ark. Fysik 23 (1963), 307-340. 